\title{
Data Routing and Spectrum Utilization Techniques in Cognitive IOT Network
}

\author{
Shailendra Kumar Tiwari ${ }^{1}$, Dr. Praveen Kumar Mannepalli ${ }^{2}$ \\ ${ }^{1}$ Research Scholar, Department Of Computer Application, LNCT University, Bhopal, Madhya Pradesh, India. \\ ${ }^{2}$ Associate Professor, Department Of Computer Science and Engineering, LNCT University, \\ Bhopal, Madhya Pradesh, India.
}

Correspondence should be addressed to Shailendra Kumar Tiwari; shailendra.t86@gmail.com

Copyright ( 2021 Made Shailendra Kumar Tiwari et al. This is an open access article distributed under the Creative Commons Attribution License, which permits unrestricted use, distribution, and reproduction in any medium, provided the original work is properly cited.

\begin{abstract}
Wireless devices increase the comfort of human life by reducing labor work and data reading accuracy. Wireless device transfer data though allotted spectrum or free available spectrum. An IOT device has limited resources to establish communication between source and destination. So paper has work on cognitive IOT network which depends on algorithms that can sense spectrum efficiently and route data. Paper has summarized various researcher work done for blank spectrum sensing in cognitive IOT network which may be co-operative or nonco-operative in nature. Further some of network routing issues were explained that were directly or indirectly depends on hardware limitation, algorithm computation, etc. Routing algorithms were also list in the work which was used by scholars to resolve different network issues.
\end{abstract}

KEYWORDS- Cognitive radio, Data Routing, Resource Utilization, Spectrum Sensing.

\section{INTRODUCTION}

With the rapid growth of technology in the field of wireless communication and embedded computing it is now possible to pack sensing, power supply, and wireless communication into a small size that of cubic centimeter and even a few cubic millimeters[1]. They can easily be attached to items such as people, food items and can even be embedded into homes and offices due to their tiny size and can be dispersed in form of a wireless network in fields or forests. Such tools prove quite useful for tracking and observing the real world. Common challenges associated with IoT-based networks are minimum delay, greater throughput, greater network lifetime, and maintaining minimum energy consumption so that better efficiency can be achieved. Due to their less size, it is difficult to maintain sufficient battery supply [3] to run such gadgets as these devices are expected to run for a longer period. Other problems with such gadgets are the limited buffer space and channel bandwidth. An event-driven approach is used to drive these sensor networks that help in establishing many-to-one communication. The nodes that are closer to the sink possess a high arrival rate hence they cause buffer overflow [4]. Due to this lifetime of the network is decreased. Many steps were taken to reduce the above problems namely cluster-based routing technique and even reactive routing techniques [5] The network is divided into several small clusters in the process of clustering. This is done to reduce the number of direct transmissions fro $3 \mathrm{~m}$ source to destination. A $\mathrm{CH}$ or cluster head is allotted who is responsible for all the activities of communication within its cluster. But still, there are two major disadvantages of clustering one is that the maintenance of $\mathrm{CH}$ is difficult in limited resources and heavy computational algorithm of the $\mathrm{CH}$. Another method is to use proactive routing which follows hop-by-hop techniques and establishes connection only when needed. The connection is established while the exchange of hello messages and a new route is established in case of loss of the older route from the routing table.

In the reactive routing process, each time a new route is designed and the new route [5] is calculated between the sender and receiver. But this causes problems such as reduced throughput and gives additional delays and also wastes the energy supply provided. Thus this process was not found suitable in real-time monitoring.

\section{SPECTRUM UTILIZATION AND COGNITIVE NETWORKS}

Spectrum utilization directly related to carrier allotted bandwidth for a blocks or packets in the network to the available bandwidth. To increase this spectrum utilization amount of data transfer should be increase, hence multi channel concept was involved. Still some of channels were unused, so cognitive radio network will sense and utilized free channel for transferring data. This increases the channel utilization.

The techniques for increasing the frequency spectrum are classified from the purpose viewpoint in [31] as

1) Increasing the number of radio channel, by narrowing the frequency spectrum technique.

2) In cellular system spatial frequency spectrum reuse was done for enhancing spectrum utilization technique.

3) Time wise frequency spectrum reuse was done in Multi channel access, TASI, delay connection service and reservation connection service.

4) Off air call setup and variable bit rate control technique reduces invalid frequency spectrum waste.

5) Information compression techniques reduce traffic without losing information. 


\section{A. Spectrum Sensing}

Here emphasis has been given to utilize the unused spectrum and allotting them to other users. Special functions are used here to find the free bandwidth for SU allocation[6]

\section{B. Spectrum Decision}

Call model spectrum is being used in this by which access has been done. And all the fine quality spectrum parameters $[7,8]$ depend on the high quality of the model.

\section{Sharing}

To maintain the quality of spectrum sensing results are taken and it has to check before its SU allocation. Quality of sensing is calculated on the basis of the signal is to noise ratio(SNR).

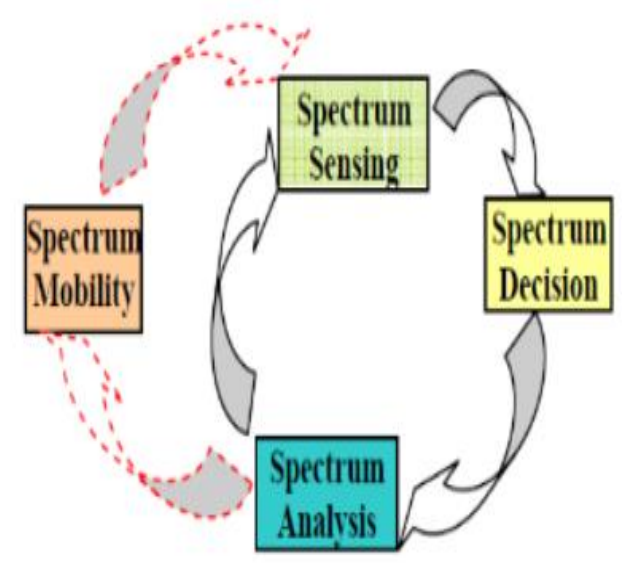

Fig. 1: Illustration of spectrum management functionalities.

\section{Spectrum quality or Handoff}

The signal establishes between the users keeps hopping from time to time to maintain the connection secure. The main motive for this hopping is to keep the connection to a more high-quality channel and this dynamic change of channels leads to find the better frequency.

\section{RELATED WORK}

This section was divided into two parts first for data routing methods proposed by various scholars. Second was spectrum sensing and optimization methods proposed by different researchers.

\section{A. Data Routing}

Aurimas Gedminas et. al. in [9] proposed a max-min algorithm for optimization of the spectrum. It was called MMAPS(Max-Min Ant P system) and it was successfully applied to ACS or Antcolony system to solve data routing problems. It was also found that this system simplifies the complex behavior of annotation and also gives a good balance of performance, scalability, and flexibility. Due to all such reasons, the P system framework has been adopted in IoT for data routing purposes. M. Rossi et. al. in [10] given that a suitable rate-distortion working point at the source nodes should be present to achieve energy-efficient routing to the transmission of information. Distributed learning algorithm was used by this one can achieve multiple-objective optimization, better channel access, solve capacity constraint problems, and distortion problems. The algorithm was based on ADMM or alternating direction method of multipliers to achieve faster convergence. Z. Magubane et. al. in [11] conducted studies on RPL (Routing Protocol for Low Power and Lossy networks) and LOADng Lightweight-ondemand AdhocDistance-vector-Routing Protocol-Next Generation). Compare their energy evaluation. The whole study was focused on power consumption while transmission of data in IoT and as a result an energy-efficient routing protocol RPL was depicted. M. Durga Devi et. al. in [12] proposed ETERNAL that is contEnT cEntRic aNd load balancing algorithm for the purpose of content transmission. The routing of related data reduces traffic and solves many problems such as reduced latency, and saves battery power consumption. And thus minimum delay with better energy efficiency was achieved.

\section{B. Spectrum Sensing}

ArunKumar and Reshma [13] introduced the Markov model to solve the problem of spectrum allocation. An algorithm was developed to solve the graph coloring problem named Banker's deadlock and it proved to provide a fair distribution of the spectrum with better efficiency. Liu et al. [14] Proposed a simulated annealing technique to determine efficient spectrum assignment. It helps to solve problems in a condition such as limited capacity, and rate requirements during traffic. His study was better than the previous migration methods. Ni et al. [15] : Uses game theory for efficient spectrum assignment in radio networks. They divided the allocation problems into non-cooperative and cooperative models. Many game theory models were designed by them and ultimately the best performance model was being implemented. Hou et al. [16] Try to maximize the rate of transfer under limited error rate constraints based on improved GA. His theory provided a notable improvement over simple GA in terms of fairness of spectrum distribution. REN HAN et. al. [17] submitted a paper based on problems with spectrum allocation in IoT. Each link in the transmission path needs to be improved to increase the transmission rate performance to achieve endto-end through output by this maximum spectrum utilization can be achieved. His paper has proposed a concurrent transmission model that showed the problems of mutual interference and competition of links in concurrent transmission. Based on the above paper a better spectrum plan for the links was formulated. His paper made an attempt to solve the non-dominated sorting algorithm to achieve multi-objective spectrum allocation.

1) Some of Major Problems Identified in the papers were:

2) Spectrum sensing was done by hard fusion techniques lead to increase collision in the network. So soft fusion should technique was in demand.

3) IOT Processing unit should be strong to execute route generation algorithms. Routing calculation should be centralized, this reduce device cost and increase energy utilization in transferring data.

4) Most of paper has assumed a dedicated spectrum channel for transferring data. Most of IOT device have undedicated channel for transmission.

5) Running a genetic algorithm leads to generate random path. Random path may decrease spectrum utilization. 


\section{ROUTING ISSUES}

A few of the challenges found in routing Issues in IoT are discussed here

1) Context Awareness: all the devices behave as actors in IoT and uses their skills to achieve fast routing by gathering information by taking quick decisions

2) Heterogeneity: Relates to the combination of various technologies in IoT that includes complex routing processes together with different devices. Every protocol has certain boundaries and so it is compulsory to build a routing protocol that follows this heterogeneity.

3) Death of Nodes: Over usage of energy usage in a network causes the death of nodes. Due to this energy hole is created that causes short jumps in the routing process and so it is essential to change the battery of nodes regularly.

4) Topological Changes: Many times the topology of the network changes for various reasons such as conservation of energy, and constraints of nodes. A hybrid routing protocol is a design to solve this problem.

5) Scalability: Relates to the wireless communication and entering and exiting of the mobile that increases or decreases the size of the network. This scalability of the network effects routing.

6) Latency: It is necessary to send the data to its destination before the data expires in a network. All such tasks need to be done within the stipulated time to achieve quality in-network services.

7) Incentive-based Routing: Every device needs to communicate with each other to achieve better routing.

8) Congestion Control: Such a problem occurs with nearly all types of networks. It exists due to an increase in traffic which causes loss of packets. To solve this problem balancing should be achieved at every node as congestion decreases the life of the network.

9) Data Security: Authentication is necessary to prevent data theft in the IoT as there are data of several different owners in an IoT.

10) Elimination of data redundancies: It is necessary to use the process of data Coalation to save the wastage of networks as IoT networks send a large amount of data.

11) Multipath routing: Load balancing and multipath routine should be followed to perform load balancing and to achieve a better lifetime of the networks and to provide reliable quality of services

12) Limited Resources: Limitation of resources and various other parameters such as power, memory, communication bandwidth, range are major problems in IoT. To overcome this problem communication should be done in a multihop fashion and multiple relay modes should be used to forward data packets to their destination. Memory and power should be optimized to their fullest in the IoT. The size of the packets is limited due to limited power and bandwidth available in the IoT so steps to conserve power have to be taken.

13) Node Deployment: For instant communication, routing node deployment should be done in sensor networks. The node deployment consists of manual and has to arrange manually in sensor nodes. They should be arranged in a random manner with the help of Adhoc routing.

\section{Data Routing TechniQues}

A protocol has to be designed which matches the traffic pattern, and power consumption to solve the limitation of the IoT system and meet the specific requirement, and use different strategies[20]. It should also be scale with the memory and performance of the IoT. Also, this routing protocol should detect and avoid one-way links to reduce power consumption. Lastly, supportingIPV6 and mobility qualities should also be present in this routing protocol. The strategy may include reactive routing and should try to have a global view of the whole network topology [21, 22]. The taxonomy of the most important routing protocol is mentioned in table- 4 .

\section{A. $R p l$}

A Distance Vector Routing Protocol was released in 2012 by IETF for lossy and low power networks. RPL creates a single path from the leaf to root which is called DODAG(Destination Oriented Directed Acyclic Graph) and thus the whole traffic is concentrated on the roots and the root decides the destination advertisement object(DAO) and handles all the DODAG information and DIS request of nodes who want to join in the network. Such RPL nodes can be stateless(keeping track of parent) or stateful(keeping track of both parents children).

\section{B. Rpl Enhancements}

Several enhancements were proposed to the basic RPL protocol. For example, P2P RPL [25] which was a point to point and reactive, RPL (P2P-RPL) that enables IPV6 router in an LLN, Enhanced-RPL for enhancing reliability, Dynamic RPL (D-RPL) [26] that was used to conduct dynamic applications in IoT network. D-RPL to improve energy efficiency and to reduce end-to-end delay, mRPL[27] was the mobile version and focuses on mobility management. mRPL have some problems related to unneeded handovers and developing unreliable connection so a better version called "Smarter-HOP" came which was denoted by mRPL++.

\section{Corpl}

In [28] it has a non-standard extension of RPL. It opportunistic data transmission to forward packets (using multiple forwarders or forwarder set) and was built for cognitive networks and uses DODAG topology. It also chooses the next hop to send packets within the network. Similar to RPL every node in DODGAJ topology sends information to its neighbors after sending the packets through DIO (DAG Information Object) messages and updates them to build further sets to forward packets.

\section{Carp}

Channel-Aware Routing Protocol [29] orCARP is likewise a nonstandard circulated steering kind of convention. It utilizes (Underwater Wireless Sensor Networks) UWSNs and its resources are giving less time in conveyance and decreased force utilization. It additionally keeps joins quality data which is determined from authentic information of fruitful exchange. The principle issue with CARP is that it doesn't reuse past information that is accumulated. An improvement of CARP was likewise evolved named ECARP [29] was created which permits sink nodes to save the past information thus it effectively diminishes the overhead issues of correspondence. 


\section{E. Aodv, Loadng, And Aodv}

Specially appointed On-Demand Distance Vector Routing or AODV [30] was a sort of jump by-bounce steering convention and was characterized by IETF in 2003. IT utilizes Route Request-(RREQ-) Route Reply-(RREP-) cycle. This cycle was started when any parcels need to go to any obscure objective. Two adaptations of AODV were presented (I) the Lightweight On-Demand Ad Hoc Distance Vector Routing Protocol-Next Generation (LOADng) and (ii) the AODVv2 which utilizes energy-mindful measurement. A lot other convention like AODV was made to lessen the impression and that suits well with dynamic organization conditions like AODVjr, LOAD(ng), AODVbis, LoWPAN-AODV, TinyAODV and NSTAODV.

\section{ISSUES RESOLVED BY DATA ROUTING TECHNIQUES}

RPL: Resolve multipath issue by use of directed acyclic graph information at root.

\section{A. D-Prl}

This technique resolves multipath issue with energy losses. Technique has improved this by use of end to end path routing and reduces energy losses.

\section{B. Corpl}

In this technique DODAG techniques was further enhanced by remembering the last neighbor node for data transfer, this reduces congestion issue in the network as knowledge of network for data routing increases.

\section{Carp}

This technique reduces the overhead issue of the packets. As most of information were save at some in between sink node.

\section{Aodv}

This technique resolved the scalability and node deployment issue with heterogeneous configuration. As ondemand path was developed before packet sending hence latency issue was also resolved.

\section{CONCLUSIONS}

This paper has described the routing protocols that can be used for communication in IOT and the open research challenges. Due to the lack of benchmarks, the protocols mentioned can only be evaluated based upon its features. These protocols must be simulated and tested in the target environment to reveal its potential implementation for Internet of Things. Paper has explained various approaches of spectrum sensing techniques proposed by different researcher of this field. It was obtained that cognitive IOT network need accurate sensing algorithm with data routing to increase the life of wireless network.

\section{REFERENCES}

[1] K. Christidis and M. Devetsikiotis, "Blockchains and Smart Contracts for the Internet of Things," in IEEE Access, vol. 4, 2016,

[2] A.Roger, T. Tsao, V. Daza, A. Lozano, M. Richardson, and M. Dohler, "A Security Threat Analysis for the Routing
Protocol for Low-Power and Lossy Networks (RPLs)," IETF RFC 7416, January 2015,

[3] J. L. Hernandez-Ramos, J. B. Bernabe and A. Skarmeta, "ARMY: architecture for a secure and privacy-aware lifecycle of smart objects in the internet of my things," in IEEE Communications Magazine, vol. 54, no. 9, 2016, pp. 28-35.

[4] M. Gomes, R. da Rosa Righi, C. A. da Costa, Internet of things scalability: analyzing the bottlenecks and proposing alternatives, in 6th International Congress on Ultra Modern Telecommunications and Control Systems and Workshops (ICUMT), 2014

[5] Khalid M Awan, Nadeem Ashraf, Muhammad Qaiser Saleem, Osama E Sheta, Kashif Naseer Qureshi, Asim Zeb, Khalid Haseeb and Ali, Safaa Sadiq "A priority-based congestion-avoidance routing protocol using IoT-based heterogeneous medical sensors for energy efficiency in healthcare wireless body area networks". International Journal of Distributed Sensor Networks, Vol. 15(6) 2019.

[6] Salahdine, Fatima. (2017). Spectrum Sensing Techniques For Cognitive Radio Networks.

[7] R. Sorayya and M. Suryanegara, "The model of spectrum sharing between a primary and two secondary operators," 2017 International Conference On Smart Technologies For Smart Nation (SmartTechCon), Bangalore, 2017, pp. 518522.

[8] D. M. Alias and Ragesh G. K, "Cognitive Radio networks: A survey," 2016 International Conference on Wireless Communications, Signal Processing and Networking (WiSPNET), Chennai, 2016, pp. 1981-1986

[9] Aurimas Gedminas, Dalius Navakauskas, Liudas Duoba. "P System Framework for Ant Colony Algorithm in IoT Data Routing". International Journal of Advanced Computer Science and Applications, Vol. 11, No. 4, 2020.

[10] M. Rossi, M. Centenaro, A. Ba, S. Eleuch, T. Erseghe and M. Zorzi, "Distributed Learning Algorithms for Optimal Data Routing in IoT Networks," in IEEE Transactions on Signal and Information Processing over Networks, vol. 6, pp. 179-195, 2020,

[11] Z. Magubane, P. Tarwireyi and M. O. Adigun, "Evaluating the Energy Efficiency of IoT Routing Protocols," 2019 International Multidisciplinary Information Technology and Engineering Conference (IMITEC), Vanderbijlpark, South Africa, 2019

[12] M. Durga Devi, K. Geetha, K. Saranyadevi. "Content Based Routing Using Information Centric Network For IoT". Procedia Computer Science, Volume 115, 2017.

[13] Arun Kumar, B. R., Reshma, C. R. "Spectrum Scheduling using Markov Chain Model in Cognitive Radio Networks", IOSR Journal of Computer Engineering (IOSR-JCE), 17(4), pp. 112-115, 2015.

[14] Liu, Z. M., Nasser, N., Hassanein, H. S. "Intelligent spectrum assignment and migration in cognitive radio network", EURASIP Journal on Wireless Communications and Networking, 2013(200), pp. 1-13, 2013.

[15] Ni, Q., Zhu, R., Wu, Z., Sun, Y., Zhou, L., Zhou, B. "Spectrum Allocation Based on Game Theory in Cognitive Radio Networks", Journal of Networks, 8(3), pp. 712-722, 2013.

[16] Hou, B., Zu, Y., Li, W., Liu, G., Ding, J. "Cognitive Radio Spectrum Allocation Strategy Based on Improved Genetic Algorithm", Communications and Network, 5, pp. 22-26, 2013.

[17] Ren Han, Yang Gao, Chunxue Wu, And Dianjie Lu. "An Effective Multi-Objective Optimization Algorithm For Spectrum Allocations In The Cognitive-Radio-Based Internet Of Things". Ieee Access March 19, 2018.

[18] A Dhumane, R Prasad, J Prasad. Routing Issues in Internet of Things: A Survey. InProceedings of the International MultiConference of Engineers and Computer Scientists. 2016; Vol. 1, 16-18. 
[19] Ravi Kumar Poluru and Shaik Naseera. "A Literature Review on Routing Strategy in the Internet of Things". Journal of Engineering Science and Technology Review 10 (5), 2017.

[20] A. Dhumane, "Routing challenges in internet of things," CSI Magazine, vol. 03, 2015.

[21] M. Talwar, "Routing techniques and protocols for internet of things: a survey," in Proceeding of the NCRIET-2015, pp. 417-423, 2015 .

[22] T. B. A. H. Prasad, "Network routing protocols in iot," International Journal of Advances in Electronics and Computer Science, vol. 4, no. 4, 2017.

[23] H. Prasad and S. Babu, "A survey on network routing protocols in internet of things (IOT)," International Journal of Computer Applications, vol. 160, no. 2, pp. 18-22, 2017.

[24] H.-S. Kim, J. Ko, D. E. Culler, and J. Paek, "Challenging the IPv6 routing protocol for low-power and lossy networks (RPL): a survey," IEEE Communications Surveys \& Tutorials, vol. 19, no. 4, pp. 2502-2525, 2017.

[25] M. Zhao, A. Kumar, P. H. Joo Chong, and R. Lu, "A comprehensive study of RPL and P2P-RPL routing protocols: Implementation, challenges and opportunities," Peer-to-Peer Networking and Applications, vol. 10, no. 5, pp. 1232-1256, 2017.

[26] H. Kharrufa, H. Al-Kashoash, Y. Al-Nidawi, M. Q. Mosquera, and A. H. Kemp, "Dynamic RPL for multi-hop routing in IoT applications," in Proceedings of the 13th Annual Conference on Wireless On-Demand Network Systems and Services (WONS '17), pp. 100-103, February 2017.

[27] A. Aijaz and A. H. Aghvami, "Cognitive machine-tomachine communications for internet-of-things: a protocol stack perspective," IEEE Internet of Things Journal, vol. 2, no. 2, pp. 103-112, 2015.

[28] S. Basagni, C. Petrioli, R. Petroccia, and D. Spaccini, "CARP: a channel-aware routing protocol for underwater acoustic wireless networks," Ad Hoc Networks, vol. 34, no. supplement C, pp. 92-104, 2015, Advances in Underwater Communications and Networks.

[29] Z. Zhou, B. Yao, R. Xing, L. Shu, and S. Bu, "E-CARP: an energy efficient routing protocol for UWSNs in the internet of underwater things," IEEE Sensors Journal, vol. 16, no. 11, pp. 4072-4082, 2015.

[30] L. R. Stojkoska and K. V. Trivodaliev, "A review of Internet of Things for smart home: Challenges and solutions," Journal of Cleaner Production, vol. 140, pp. 1454-1464, 2017.

[31] H. Sekiguchi, H. Ishikawa, M. Koyama and H. Sawada, "Techniques for increasing frequency spectrum utilization in mobile radio communication system," 35th IEEE Vehicular Technology Conference, Boulder, CO, USA, 1985, pp. 2631

\section{ABOUT THE AUTHORS}

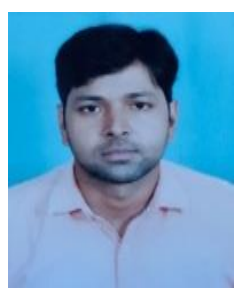

Shailendra Kumar Tiwari, Research Scholar, Department of Computer Application, LNCT University, Bhopal, Madhya Pradesh, India.

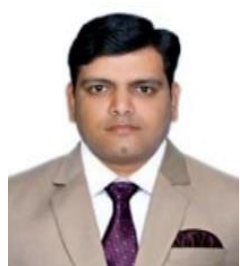

Dr. Praveen Kumar Mannepalli, Associate Professor, Department of Computer Science and Engineering, LNCT University, Bhopal, Madhya Pradesh, India 